\title{
Large eddy simulation of a Mach 0.9 turbulent jet
}

\author{
By G. A. Brès $\dagger$, P. Jordan $\ddagger$, T. Colonius $\uparrow$, M. Le Rallic $\ddagger$, V. Jaunet $\ddagger$ \\ AND S. K. Lele
}

Large eddy simulations of an isothermal Mach 0.9 jet $\left(\operatorname{Re}=10^{6}\right)$ issued from a convergent-straight nozzle are performed using the compressible flow solver CharLES. The flow configuration and operating conditions match the companion experiment conducted at the PPRIME Institute, Poitiers. To replicate the effects of the boundary layer trip present in the experiment and to ensure a turbulent jet, localized adaptive mesh refinement, synthetic turbulence, and wall modeling are used inside the nozzle. This leads to fully turbulent nozzle-exit boundary layers and results in significant improvements for the flow field and sound predictions, compared to those obtained from the typical approach based on laminar flow assumption in the nozzle. The far-field noise spectra now match the experimental measurements to within $0.5 \mathrm{~dB}$ for relevant angles and frequencies. As a next step toward better understanding of turbulent jet noise, the large database collected during the simulation is currently being used for reduced order modeling and wavepacket analysis (Jordan et al. 2014).

\section{Introduction}

To improve understanding and modeling of the turbulent sources of sound in subsonic jets, extensive experimental and numerical databases were generated for a turbulent Mach 0.9 jet. The experiments conducted at PPRIME Institute include characterization of the nozzle-exit boundary layers, flow field PIV, and both near-field and far-field pressure measurements on large microphone arrays. As part of the CTR summer program, a companion large eddy simulation (LES) was performed using the compressible flow solver CharLES developed at Cascade Technologies. One of the challenging aspects of the present configuration is that transition to turbulence is forced in the experiment using a boundary layer trip inside the nozzle. From past experimental (Bradshaw et al. 1964; Hill et al. 1976; Bridges \& Hussain 1987) and numerical studies (Bogey \& Bailly 2010; Bogey et al. 2012), the state of the nozzle-exit boundary layer is known to be a key parameter for the flow development and noise characteristics of a jet. However, because of the computational cost of simulating high Reynolds number wall-driven turbulence, the nozzle boundary layer is typically assumed to be laminar or weakly disturbed in most jet simulations. This approach often leads to enhanced laminar-to-turbulent shear-layer transition and increased noise due to vortex pairing.

The present simulations leveraged recent research efforts sponsored by ONR and NAVAIR focusing on this important aspect of the jet noise problem: the modeling of the nozzle interior flow and its effects on the nozzle-exit boundary layer, the jet plume, and ultimately the acoustic field (Brès et al. 2013, 2014). Here, localized adaptive mesh refinement, synthetic turbulence, and wall modeling are used inside the nozzle to ensure

$\dagger$ Cascade Technologies Inc.

$\ddagger$ Institute PPRIME, CNRS - Univ. Poitiers, ENSMA, France

I Department of Mechanical Engineering, California Institute of Technology 

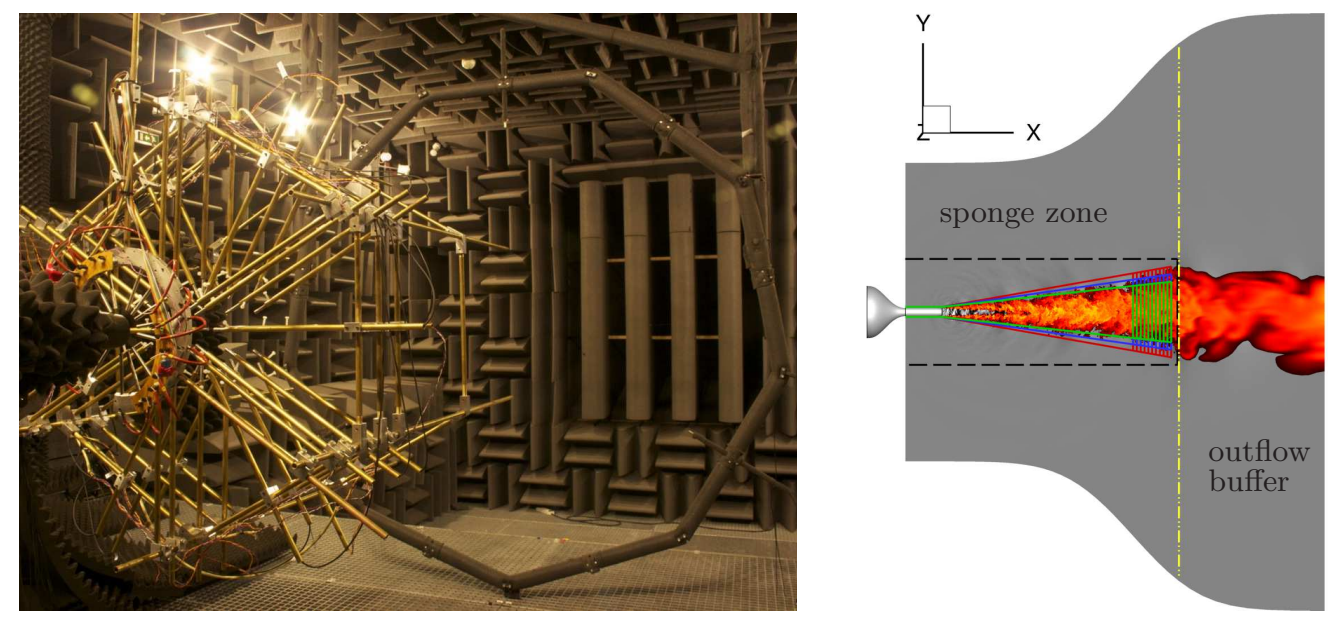

Figure 1. (a) Anechoï jet-noise facility; (b) Schematic of the flow configuration and numerical setup (color online)

fully turbulent profiles at the nozzle exit. These modeling approaches, as well as the experimental configuration and the numerical setup, are briefly reviewed in Section 2. Then, in Section 3, results from a series of preliminary large eddy simulations are discussed to highlight the improvements associated with the different modeling applied inside the nozzle. Details of the main LES database generated during the CTR summer program are also presented, including comparisons with experimental measurements.

\section{Flow configuration and numerical methods}

\subsection{Experimental setup}

The study focuses on an isothermal Mach 0.9 jet issued from contoured convergentstraight nozzle of exit diameter $D=50 \mathrm{~mm}$. The experiments were performed in the anechoïc jet-noise facility of the PPRIME Institute at the Centre d'Études Aérodynamiques et Thermiques (CEAT), Poitiers, France (see Figure 1(a)). The experimental diagnostics included hot-wire and laser doppler velocimetry (LDV) systems for measurement of the exit velocity profiles, particle image velocimetry (PIV) for more extensive measurement of the jet plume, a 48-microphone near-field cage array for the eduction of wavepacket signatures, and an axially traversable, 18-microphone, azimuthal array providing measurements of the sound field on a cylindrical surface of radius $r / D=14.3$. Pressure measurements are also performed in the far field at a constant distance of $50 D$ from the nozzle exit using a single microphone every $10^{\circ}$, from inlet angle $90^{\circ}$ (i.e., normal to nozzle-exit plane) to $160^{\circ}$ (i.e., downstream of nozzle exit)

Comparisons presented in this report are, for the sake of brevity, limited to the exit velocity profiles and acoustic measurements. A more comprehensive comparison will be presented in future publications.

\subsection{Numerical setup}

In this work, the jet is investigated with the high-fidelity LES framework developed at Cascade Technologies. The framework is composed of the pre-processing mesh adaptation tool Adapt, the compressible flow solver CharLES, and post-processing tools for far-field noise predictions based on an efficient massively-parallel implementation (Brès 


\begin{tabular}{|c|c|c|c|c|c|c|c|}
\hline Case name & $\begin{array}{l}\text { Mesh size } \\
\left(10^{6} \text { cells }\right)\end{array}$ & $\left|\begin{array}{c}\mathrm{BL} \\
\text { refine- } \\
\text { ment }\end{array}\right|$ & $\begin{array}{c}\text { Synthetic } \\
\text { turbulence } \\
u_{\text {trip }}^{\prime} / u_{\tau}\end{array}$ & $\left|\begin{array}{c}\text { Wall } \\
\text { model }\end{array}\right|$ & $d t c_{\infty} / D$ & $t_{\text {sim }} c_{\infty} / D$ & $\begin{array}{l}\text { Database } \\
\text { sampling } \\
\Delta t c_{\infty} / D\end{array}$ \\
\hline
\end{tabular}

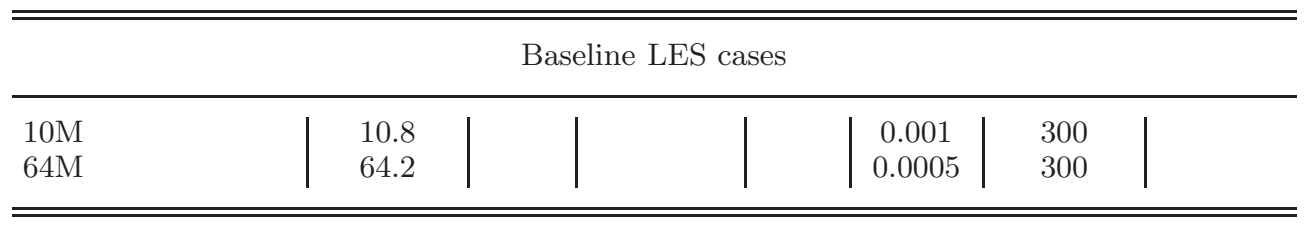

LES cases with nozzle interior flow modeling

\begin{tabular}{l|c|c|c|c|c|c|c}
\hline BL16M & 15.9 & $\times$ & & & 0.001 & 300 & \\
BL16M_Turb2 & 15.9 & $\times$ & 2 & & 0.001 & 300 & \\
BL16M_Turb & 15.9 & $\times$ & 0.8 & & 0.001 & 300 & \\
BL16M_WM & 15.9 & $\times$ & & $\times$ & 0.001 & 300 & \\
BL16M_WM_Turb2 & 15.9 & $\times$ & 2 & $\times$ & 0.001 & 300 & \\
BL16M_WM_Turb & 15.9 & $\times$ & 0.8 & $\times$ & 0.001 & 2000 & 0.2 \\
BL69M_WM_Turb & 69.0 & $\times$ & 0.8 & $\times$ & 0.0005 & 300 & 0.2 \\
BL432M_WM_Turb & 432.2 & $\times$ & 0.8 & $\times$ & 0.00025 & 250 & 0.2 \\
\hline
\end{tabular}

TABLE 1. Simulation parameters of the different LES performed (BL16M_WM_Turb: database used during the CTR summer program). For all cases, the sampling period of the data recording on the FW-H surface is $\Delta t_{F W H} c_{\infty} / D=0.05$.

et al. 2012a) of the frequency-domain permeable formulation (Lockard 2000) of the Ffowcs Williams \& Hawkings (1969) (FW-H) equation.

The numerical setup, the FW-H surfaces used to compute the far-field noise (see Figure $1(b)$ ), and the baseline methodologies are similar to previous jet studies with the flow solver CharLES (Brès et al. 2012b,a, 2013, 2014; Nichols et al. 2012, 2013). The nozzle pressure ratio and nozzle temperature ratio are $N P R=P_{t} / P_{\infty}=1.7$ and $N T R=T_{t} / T_{\infty}=1.15$, respectively, and match the experimental conditions. Here, the subscript $t$ and $\infty$ refer to the stagnation (total) property and free-stream (ambient) quantity, respectively. The jet is isothermal $\left(T_{j} / T_{\infty}=1.0\right)$, and the jet Mach number is $\mathrm{M}_{j}=U_{j} / c_{j}=0.9$, where $U_{j}$ is the mean (time-averaged) streamwise jet velocity and the subscript $j$ refer to the jet properties. For both experiment and simulation, the Reynolds number is $\operatorname{Re}_{j}=\rho_{j} U_{j} D / \mu_{j} \approx 1 \times 10^{6}$.

The round nozzle geometry (with exit centered at $(0,0,0)$ ) is explicitly included in the axisymmetric computational domain, which extends from approximately $-10 D$ to $50 D$ in the streamwise $(\mathrm{x})$ direction and flares in the radial direction from $20 D$ to $40 D$ (Figure $1(b)$ ). Note that a very slow coflow at Mach number $\mathrm{M}_{\infty}=0.009$ is imposed outside the nozzle in the simulation $\left(\mathrm{M}_{\infty}=0\right.$ in the experiment), to prevent any spurious recirculation and facilitate flow entrainment. Sponge layers and damping functions are applied to avoid spurious reflections at the boundary of the computational domain. The Vreman (2004) sub-grid model is used to account for the physical effects of the unresolved turbulence on the resolved flow. An extension to the digital filtering technique (Klein et al. 2003) for the generation of synthetic turbulence on unstructured grids is currently being developed by Cascade Technologies in collaboration with Stanford University. When 


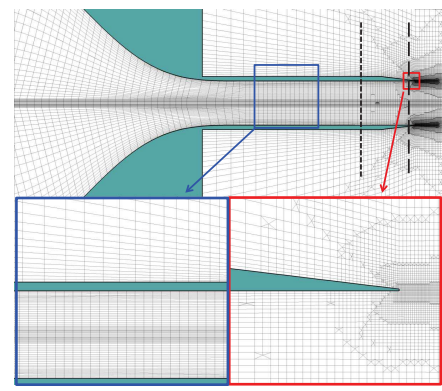

(a) Baseline $10 \mathrm{M}$ case

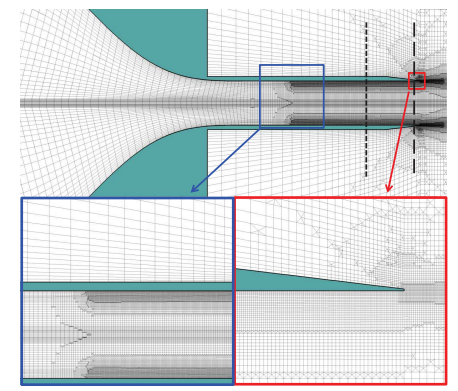

(b) BL16M case
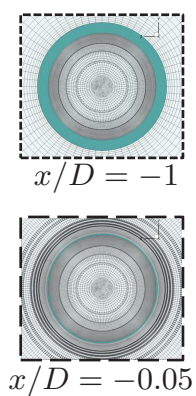

$\bar{x} \bar{D}=-0.05$

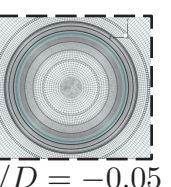
FIGURE 2 . Mesh inside the nozzle $(a)$ without and $(b)$ with boundary layer grid isotropic refine-
ment. The blue and red boxes are the zoomed-in views near the boundary layer trip and the nozzle tip, respectively (color online). Cross-sections of the grid inside the nozzle at $x / D=-1$ (short-dash line and box) and near the nozzle exit at $x / D=-0.05$ (long-dash line and box) are also shown.

active, synthetic turbulence boundary conditions are used to model the boundary layer trip present in the experiment at $-2.8<x / D<-2.5$ on the internal nozzle surfaces. Based on an estimate of the wall friction velocity $u_{\tau}$ from the baseline LES calculation at the trip location, synthetic turbulence fluctuations were introduced with a prescribed amplitude, $u_{\text {trip }}^{\prime} / u_{\tau} \sim A$. In the baseline simulations, all the solid surfaces are treated as no-slip adiabatic wall. When active, the wall model by Bodart \& Larsson (2011) is applied inside the nozzle, in the straight pipe section between the boundary layer trip and the nozzle exit.

Table 1 lists the settings and parameters for each LES run considered, including the total simulation time (after the initial transient was removed) $t_{\text {sim }} c_{\infty} / D$, and the data sampling period $\Delta t c_{\infty} / D$ for the cases where the LES database was collected. The main case used during the CTR summer program is BL16M_WM_Turb.

\subsection{Mesh adaptation and refinement}

The same grid adaptation approach used for previous jet studies by Brès et al. (2012b, 2013,2014 ) is applied to the present configuration. The starting point is a coarse skeletal grid with a paved core, containing about 0.4 million control volumes. Several embedded zones of refinement are then defined by the user and enforced by the Adapt tool. The main refinement zones correspond to the bulk of the mesh (containing the jet plume and fully enclosing the FW-H surfaces used for the far-field noise predictions), the jet potential core, and near-nozzle exit.

For the baseline cases, two grids were generated: a coarse mesh contained approximately 10 million unstructured control volumes (see Figure 2(a)), and a standard mesh with 64 million cells, by doubling the resolution in the each refinement zones. Note that for these cases, there is no specific near-wall or nozzle interior refinement, and both grids have exactly the same coarse resolution inside the nozzle.

In contrast, for the simulations involving enhanced modeling of the interior nozzle flow, it can be anticipated that some amount of mesh isotropy is also needed inside the nozzle to resolve the large-scale three-dimensional turbulent structures associated with the internal boundary layers. Therefore, isotropic refinement is added to the previous adaptation strategy and applied from the start of the boundary layer trip at $x / D=-2.8$ to the nozzle exit at $x / D=0$, for a constant distance $0.085 D$ from the nozzle wall and with target length scale equal to $0.0075 D$. The distance was chosen based on the 


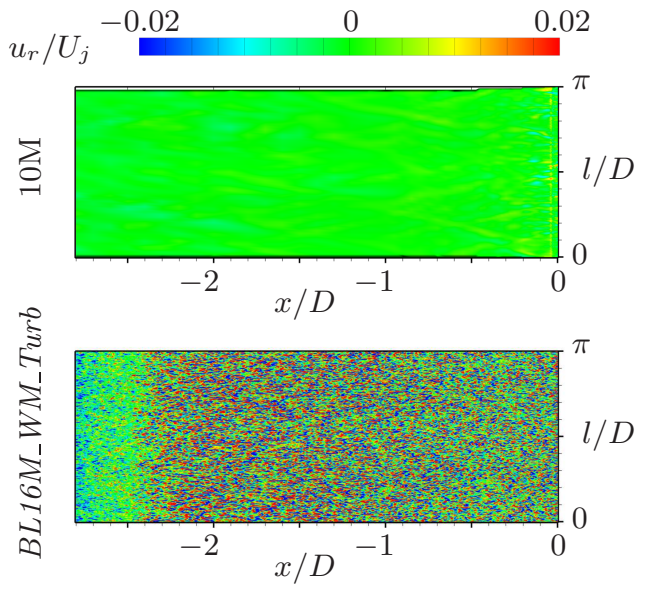

(a) Wall-normal velocity

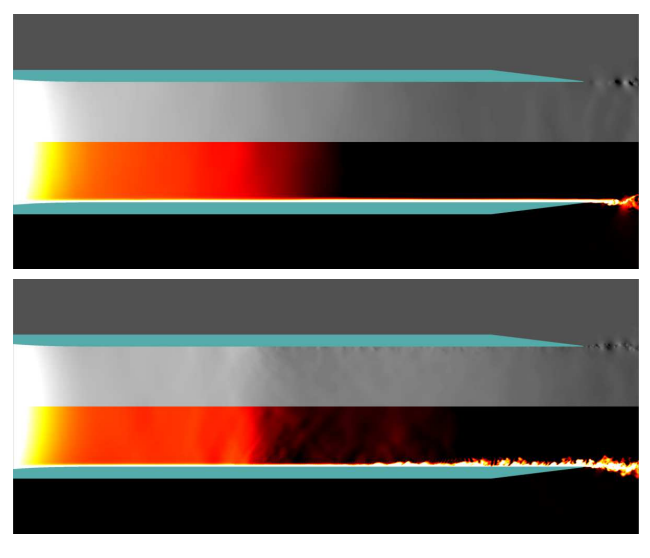

(b) Pressure and temperature

FiguRE 3. Zoom-in view of the instantaneous flow field inside the nozzle, for the baseline 10M case (top row) and the LES cases with nozzle interior modeling BL16M_WM_Turb (bottom row): (a) Wall-normal velocity $u_{r} / U_{j}$ in the first cell near the (unrolled) nozzle interior surface. Recall that the synthetic turbulence is applied for $-2.8 \leq x / D \leq-2.5$, when active; $(b)$ pressure (top half - gray scale) and temperature field (bottom half - red scale) in the mid-section plane $(z=0)$ inside the nozzle (color online).

experimental nozzle-exit boundary layer thickness, $\delta_{\exp } / D \approx 0.08$, and the length scale was chosen to yield about 10-20 LES cells in the boundary layer. The adapted grids with boundary layer refinement now contain approximately 16 million (see Figure 2(b)), 69 million and 432 million cells, for the coarse, standard and refined cases, respectively. This isotropic refinement strategy effectively results in about 1050 cells in the azimuthal direction near the nozzle surface.

\section{Parametric study and LES database}

In preliminary work prior to the CTR summer program, a systematic parametric study of the separate and combined effects of different modeling within the nozzle interior was conducted on the coarse mesh, focusing on localized adaptive mesh refinement inside the nozzle, application of synthetic turbulence and wall modeling. To conciseness, the results presented in most of the figures are limited to the baseline $10 \mathrm{M}$ case and the case with full nozzle interior modeling BL16M_WM_Turb. However, the complete conclusions of the parametric studies and the main results leading to the generation of the large LES database for wavepacket analysis are summarized in the next two sections. To provide consistent comparisons, the same total simulation time $t_{\text {sim }} D / c_{\infty}=300$ was used for the computation of the flow statistics and far-field noise spectra presented here.

\subsection{Effects of nozzle interior modeling on flow field results}

Figure 3 shows the comparison of the instantaneous nozzle interior flow with and without modeling. More quantitative comparisons are presented in Figures 4 and 5 , for the nozzleexit profiles and the velocity statistics along the lipline, respectively.

The first conclusion is that all the simulations with isotropic refinement of the boundary layer mesh now display small-scale three-dimensional turbulent structures in the boundary layer inside the nozzle. Depending on the additional modeling applied inside 


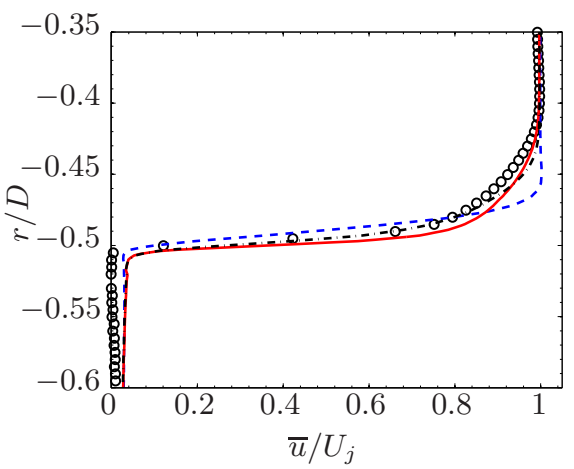

(a) Time-averaged streamwise velocity

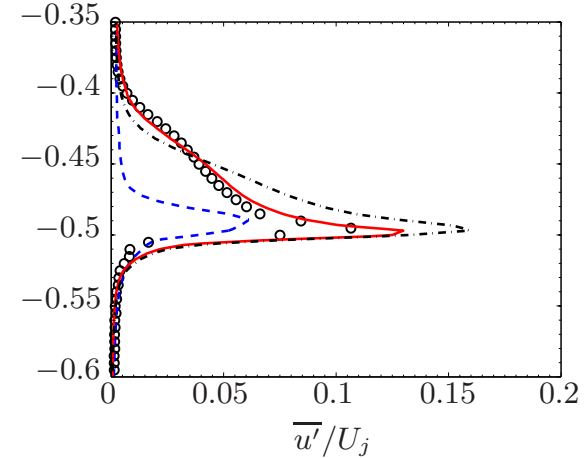

(b) RMS of streamwise velocity

Figure 4. Nozzle-exit boundary layer profiles from experiment ( o ), baseline case 10M ( ---- ), and with modeling BL16M ( - - $)$ ) and BL16M_WM_Turb ( $(-)$ (color online).

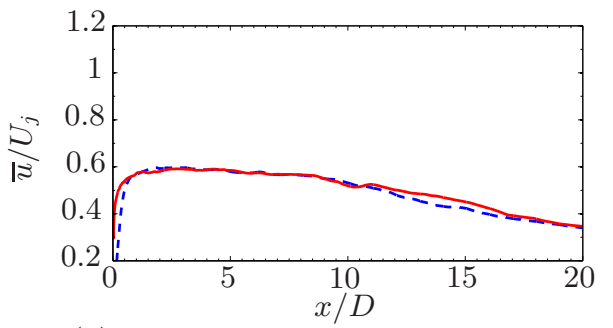

(a) Time-averaged streamwise velocity

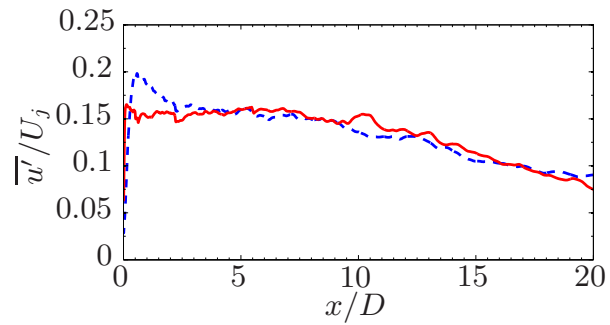

(b) RMS of streamwise velocity

FIgURE 5. Streamwise velocity statistics along the lipline for the baseline case 10M ( ---- ) and with modeling BL16M_WM_Turb ( - ) (color online).

the nozzle (i.e., synthetic turbulence, wall modeling, both, or none), the details of the development of the turbulence inside the nozzle are different. However, the internal flow field within the last 1D from the nozzle exit look similar in all cases, much like the exit profiles. All the nozzle-exit boundary layers now exhibit turbulent mean and RMS velocity profiles, with much larger fluctuation levels near the wall than in the baseline 10M case with the thin laminar boundary layer. Overall, the grid adaptation has the most significant impact on the nozzle interior flow field for the present configuration.

Second, the effect of the wall model is also clearly visible and largely beneficial. While the nozzle-exit RMS levels are over-predicted compared to experiment for cases BL16M (see Figure 4(b)) and BL16M_Turb, the cases with wall modeling show less of an overshoot and better agreement with the measurements.

Finally, modeling of the experimental trip with synthetic turbulence also slightly improved the interior nozzle flow predictions, but overall had a more limited impact than the two previous items. Two different levels of amplitudes for the synthetic turbulence were tested (see Table 1), and the results show that the nozzle-exit boundary layer and turbulence were largely independent of these input parameters.

The velocity statistics along the lipline in Figure 5 also shows improved results for the LES cases with nozzle interior modeling, consistent with previous observations. The most drastic change can be observed in the velocity RMS along the lipline in Figure 5(b) where the fluctuation overshoot around $x=0.5 D$ (related to the shear layer laminar to turbulent transition) is nearly completely removed with modeling. 

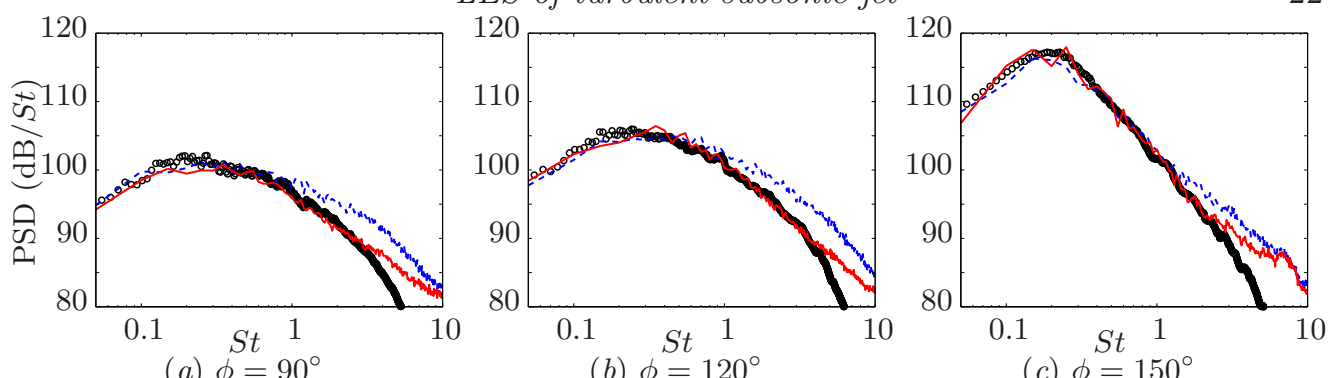

Figure 6. Comparison of the noise spectra from experiment ( $\circ)$, baseline case 10M ( ---- ) and with modeling BL16M_WM_Turb ( - ) at 50D from the nozzle exit for selected inlet angles $\phi$ (color online).

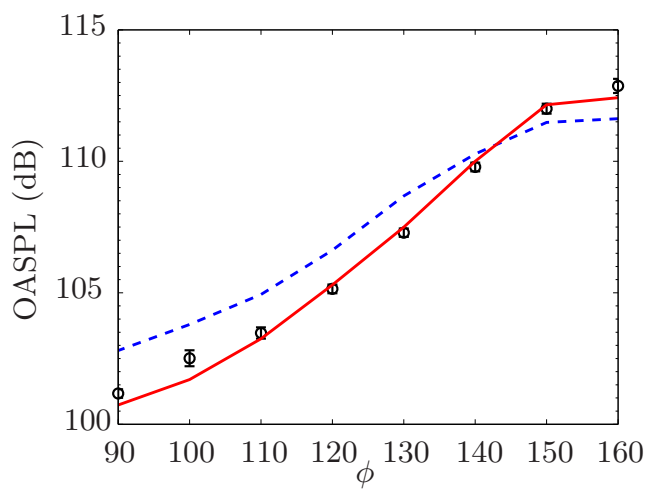

Figure 7. Overall sound pressure level from experiment ( ० ), baseline case 10M (---- ) and with modeling BL16M_WM_Turb ( $\_$) (color online).

\subsection{Effects of nozzle interior modeling on far-field acoustic results}

Figures 6 and 7 show the comparisons between experiment, the baseline case and case with nozzle interior modeling, for the PSD spectra at the different experimental microphones, and the OASPL directivity, respectively.

Like the flow field results discussed in the previous section, the grid adaptation has the most significant impact on far-field noise predictions. The over-prediction (i.e., the blue dashed curve in Figures 6) observed at high frequency for all inlet angles and all baseline cases, independently of the refinement in the jet plume, is eliminated. Excellent agreement with experimental measurements is obtained for all angles and frequencies up to $\mathrm{St} \approx 4$ on the present coarse mesh. The resulting OASPL directivity curve in Figure 7 now lies within experimental uncertainty, with less than a $0.5 \mathrm{~dB}$ difference for most angles.

\subsection{LES database}

Based on the preliminary LES study, the case BL16M_WM_Turb with isotropic refinement of the boundary layer mesh inside the nozzle, wall modeling and synthetic turbulence was down-selected to generate the long LES database for the CTR summer program. The total simulation time $t_{\text {sim }} D / c_{\infty}$ was extended to 2000 and the full LES flow field in primitive variable $(\rho, P, u, v, w)$ was collected every $\Delta t D / c_{\infty}=0.2$ (see Table 1 ). Complete validation of the noise predictions is presented in Figure 8 for all the near-field and far-field experimental microphones. Additional simulations for the same configuration 


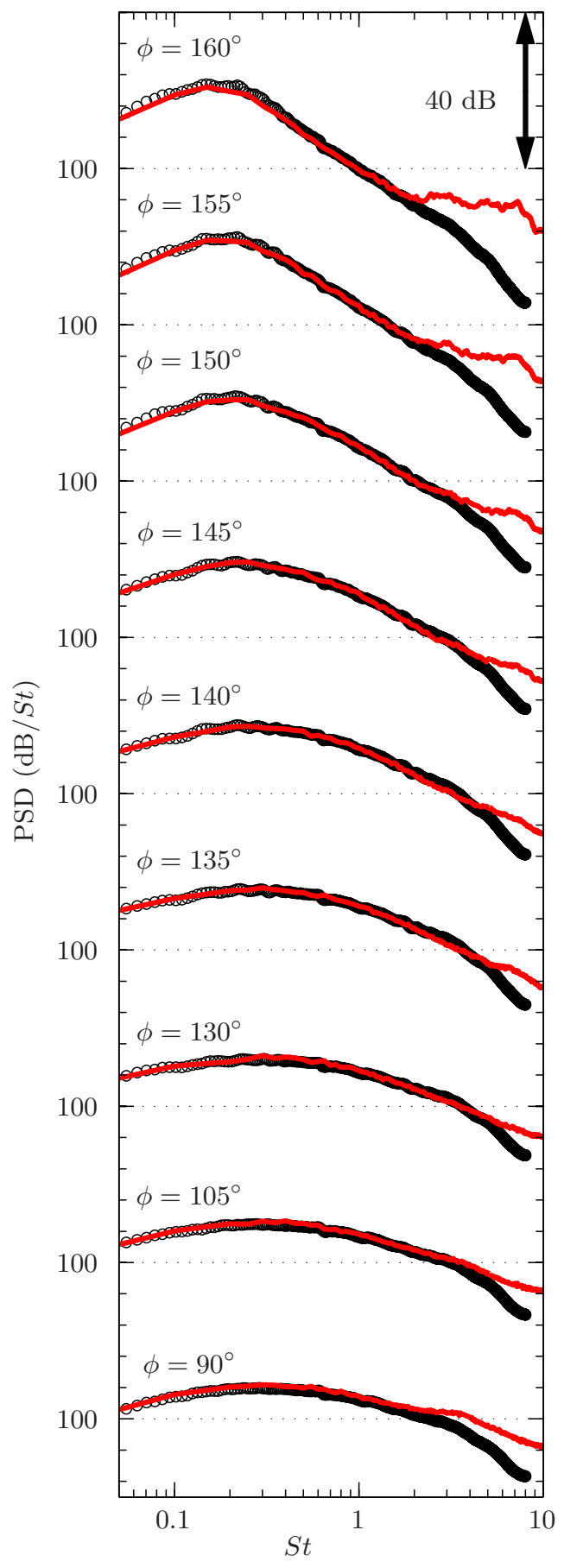

(a) Cylindrical array of radius $14.3 \mathrm{D}$

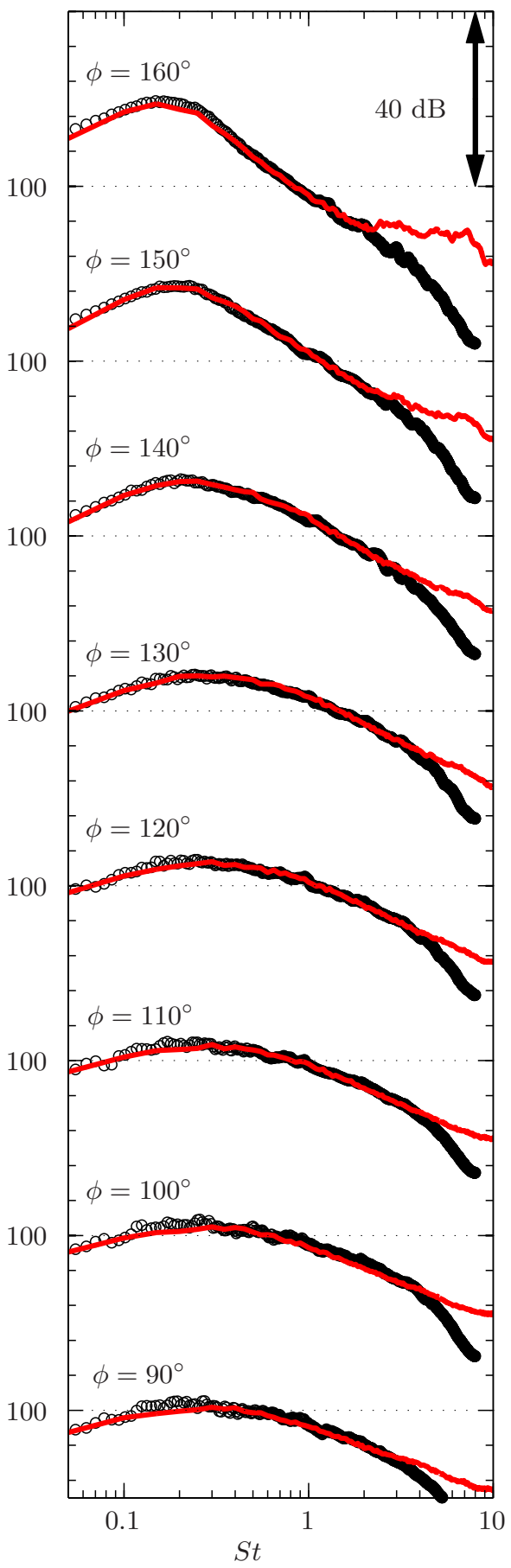

(b) Far-field array at constant $r / D=50$

FiguRE 8. Comparison of the noise spectra from experiment ( $\circ$ ) and LES case with modeling BL16M_WM_Turb ( — $)$ for all the experimental microphones (color online). 
and numerical setup have been performed as well on the standard mesh (i.e., 69M cells) and refined mesh (i.e., 432M cells). Postprocessing and analysis on the resulting LES databases are currently ongoing, including further comparisons with measurements from the recent experimental campaign.

\section{Conclusions}

The focus of the present project was the generation of a high-fidelity jet database for wavepacket analysis. Large eddy simulations of a isothermal Mach 0.9 jet $(\operatorname{Re} \approx$ $10^{6}$ ) issued from contoured convergent-straight nozzle were performed, using the LES framework developed at Cascade Technologies.

Particular care was taken to ensure a fully turbulent jet and improve the nozzle interior unsteady flow modeling, seamlessly coupled with high-fidelity predictions of the jet plume and radiated noise. In preliminary work, a systematic parametric study of the effects of different modeling within the nozzle interior was conducted, focusing on localized adaptive mesh refinement inside the nozzle, application of synthetic turbulence, and wall modeling. Overall, the results show significant improvement for both flow field and noise predictions when modeling inside the nozzle was applied, compared to the typical approach based on coarse resolution in nozzle and laminar flow assumption commonly used in most jet simulations. With modeling, the nozzle-exit velocity statistics now exhibit fully turbulent profiles similar to the experimental data, and the far-field noise spectra now more closely match the measurements for all angles and most relevant frequencies.

While previous attempts were made to simulate initially turbulent jets (Uzun \& Hussaini 2007; Bogey et al. 2008), this is the first time, to our knowledge, that a Mach 0.9 jet with fully turbulent nozzle-exit boundary layer has been successfully simulated, including flow field and far-field noise predictions. The resulting LES databases are currently being postprocessed and mined extensively to understand and model jet-noise source mechanisms (Jordan et al. 2014).

\section{Acknowledgments}

The preliminary LES studies were supported by a NAVAIR SBIR grant managed by Dr. John T. Spyropoulos. The simulations were performed as part of the DoD HPCMP Challenge Project entitled Large Eddy Simulations of Supersonic Jet Noise and Combustor \& Augmentor Spray Atomization. The main calculations were carried out on CRAY XE6 machines at DoD supercomputer facilities in ERDC DSRC.

\section{REFERENCES}

Bodart, J. \& LARsson, J. 2011 Wall-modeled large eddy simulation in complex geometries with application to high-lift devices. Annual Research Briefs, Center for Turbulence Research, Stanford University. pp. 37-48.

Bogey, C. \& BAILly, C. 2010 Influence of nozzle-exit boundary-layer conditions on the flow and acoustic fields of initially laminar jets. J. Fluid Mech. 663, 507-538.

Bogey, C., Barré, S. \& Bailly, C. 2008 Direct computation of the noise generated by subsonic jets originating from a straight pipe nozzle. Int. J. Aeroacoust. 7, 1-21.

Bogey, C., Mardsen, O. \& Bailly, C. 2012 Influence of initial turbulence level on the flow and sound fields of a subsonic jet at a diameter-based Reynolds number of $10^{5}$. J. Fluid Mech. 701, 352-385. 
Bradshaw, P., Ferriss, D. H. \& Johnson, R. F. 1964 Turbulence in the noiseproducing region of a circular jet. J. Fluid Mech. 7, 591-624.

Brès, G. A., Bose, S. T., Ham, F. E. \& Lele, S. K. 2014 Unstructured large eddy simulations for nozzle interior flow modeling and jet noise predictions. AIAA Paper 2014-2601.

Brès, G. A., Ham, F. E., Nichols, J. W. \& Lele, S. K. 2013 Nozzle wall modeling in unstructured large eddy simulations for hot supersonic jet predictions. AIAA Paper 2013-2142.

Brès, G. A., Nichols, J. W., Lele, S. K. \& Ham, F. E. $2012 a$ Towards best practices for jet noise predictions with unstructured large eddy simulations. AIAA Paper 2012-2965.

Brès, G. A., Nichols, J. W., Lele, S. K., Ham, F. E., Schlinker, R. H., Reba, R. A. \& Simonich, J. $2012 b$ Unstructured large eddy simulation of a hot supersonic over-expanded jet with chevrons. AIAA Paper 2012-2213.

Bridges, J. E. \& Hussain, A. K. M. F. 1987 Roles of initial conditions and vortex pairing in jet noise. J. Sound Vib. 117, 289-331.

Ffowcs Williams, J. E. \& Hawkings, D. L. 1969 Sound generation by turbulence and surfaces in arbitrary motion. Phil. Trans. R. Soc. Lond. A 264, 321-342.

Hill, G., Jenkins, R. C. \& Gilbert, B. L. 1976 Effects of the initial boundary-layer state on turbulent jet mixing. AIAA J. 14, 1513-1514.

Jordan, P., Colonius, T., Brès, G. A., Zhang, M., Towne, A. \& Lele, S. K. 2014 Modeling intermittent wave packets and their radiated sound in a turbulent jet. Proceedings of the Summer Program, Center for Turbulence Research, Stanford University, pp. 241-249.

Klein, M., SAdiki, A. \& Janicka, J. 2003 A digital filter based generation of inflow data for spatially developing direct numerical or large eddy simulations. J. Comput. Phys. 186, 652-665.

LockARD, D. P. 2000 An efficient, two-dimensional implementation of the Ffowcs Williams and Hawkings equation. J. Sound Vib. 229, 897-911.

Nichols, J. W., Lele, S. K., Moin, P., Ham, F. E. \& Bridges, J. E. 2012 Largeeddy simulation for supersonic rectangular jet noise prediction: effects of chevrons. AIAA Paper 2012-2212.

Nichols, J. W., Lele, S. K. \& Spyropoulos, J. T. 2013 The source of crackle noise in heated supersonic jets. AIAA Paper 2013-2197.

Uzun, A. \& Hussaini, Y. M. 2007 Investigation of high frequency noise generation in the near-nozzle region of a jet using large eddy simulation. Theor. Comput. Fluid Dyn. 21, 291-321.

VReman, A. 2004 An eddy-viscosity subgrid-scale model for turbulent shear flow: Algebraic theory and applications. Phys. Fluids 16, 3570. 